\title{
Promoção da Saúde e Cultura Cidadã Envolvendo uma Abordagem Oftalmológica em Escolares na Colônia Antônio Aleixo (CAA), Manaus-AM: Uma Experiência no Ensino Médico*
}

PALAVRAS-CHAVE:

- Cultura;

- Promoção da Saúde;

- Educação Médica;

- Modelos Educacionais;

- Saúde Ocular.

KEY-WORDS:

- Culture;

- Health Promotion;

- Education, Medical;

- Models, Educational;

- Eye Health.

Recebido em: 04/06/2004

Reencaminhado em: 28/10/2004 Reencaminhado em: 25/04/2006 Aprovado em: 29/06/2006

$87 \mid \begin{aligned} & \text { REVISTA BRASILEIRA DE EDUCAÇÃO MÉDICA } \\ & 30 \text { (2) : } 87-92 ; 2006\end{aligned}$

\author{
Promotion of health and Citizenship Involving \\ an Ophtalmic Approach to school children in \\ Colônia Antônio Aleixo (CAA), Manaus-AM: \\ An Experience in Medical Education
}

Jefferson Augusto Santana Ribeiro ${ }^{1}$ Alexandre dos Santos Saraiva ${ }^{1}$ André Luís Araújo ${ }^{1}$ Mennabarreto Segadilha França²

\begin{abstract}
RESUMO
Objetivou-se promover a capacitação de alunos de Medicina da disciplina de Saúde Coletiva da Universidade Federal do Amazonas e comunidade para o desenvolvimento de uma cultura cidadã dentro do paradigma socioecológico, identificando-se os problemas oftalmológicos nos escolares da comunidade. Foram realizadas visitas domiciliares semanais e discutidas questões relevantes para a saúde dos moradores e problemas da localidade, na CAA, bairro periférico de Manaus. Escolares de 4 a 14 anos dessa comunidade foram triados na avaliação oftalmológica (AO), com encaminhamento dos casos ao especialista. Os problemas encontrados - desemprego, saneamento básico, desconhecimento das leis e baixo grau de escolaridade - motivaram reuniões entre moradores, acadêmicos e profissionais de setores relacionados. De 133 crianças avaliadas na AO, uma precisou de tratamento. Constatou-se que o acadêmico de Medicina inserido na realidade que o cerca é capaz de se tornar um profissional mais ativo na sociedade na luta por uma localidade saudável/cidade saudável, capacitando a comunidade para suas lutas sociais em busca de melhor qualidade de vida e exercício pleno da cidadania. A prevalência de problemas oftalmológicos nos escolares da comunidade foi inferior à observada na literatura.
\end{abstract}

\begin{abstract}
The objective of this study was to capacitate medical students of the Public Health course of the Federal University of Amazonas and to help the community developing a citizenship-based culture within a socio-ecologic paradigm, identifying ophthalmic problems in school children of the community. Questions important for the health of the community as well as local problems of $C A A$, a neighborhood in the outskirts of the city of Manaus, were discussed in weekly household visits. School children between 4 and 14 years of age were submitted to an ophthalmic examination (OE) and the detected cases were transferred to a specialist. Problems such as unemployment, deficient basic sanitation, ignorance of the laws and low educational level were detected and motivated meetings with residents, medical students and professionals of related areas. OE was performed in 133 children. Only one needed treatment. It was verified that the
\end{abstract}


contact with real life is able to turn the medical student into a socially active professional fighting for a healthy place/healthy city and helping the community to fight its social battles in search of a better quality of life and full exercise of citizenship. The prevalence of ophthalmic problems in the community's school children was found to be lower than observed in the literature.

\section{JUSTIFICATIVA}

O distanciamento do aluno da realidade que o cerca é notório no modelo pedagógico vigente na maioria das escolas médicas, nas quais o ensino é estruturado a partir da medicina flexneriana, prevalecendo um projeto acadêmico-cêntrico, com dicotomia dos espaços acadêmicos interno e externo ${ }^{1}$. Busca-se, então, um novo modelo pedagógico, baseado em um novo paradigma (paradigma socioecológico), a medicina comunitária. Este novo modelo deve promover a inserção precoce do aluno na realidade da comunidade, rompendo com os limites dos muros da academia, e com uma visão mais ampla do processo saúde-doença, requisitos necessários para que se prepare o profissional de saúde a fim de que este seja agente de mudanças na comunidade, de fortalecimento dos grupos locais na luta por uma localidade saudável/cidade saudável e qualidade de vida. A relação academia-comunidade no novo modelo pedagógico permite que a academia supere seu isolamento e se integre "com os serviços e com as comunidades num processo de inserção na realidade" 1 , ou "cair na vida", segundo Mendes ${ }^{1}$.

O conceito de Promoção da Saúde, definido na Carta de Ottawa (Canadá, 1986) como “o processo de capacitação da comunidade para atuar na melhoria da sua qualidade de vida e saúde, incluindo maior participação no controle deste processo"2, demanda uma nova visão em saúde coletiva, "cuja abordagem norteia caminhos pelos quais população, grupos e indivíduos no exercício da cidadania deverão seguir atuando e participando ativamente das políticas públicas do setor"3. No Brasil, Mello et al. ${ }^{3}$ e Mello ${ }^{4}$ observam que, embora muito tenha se discutido sobre Promoção da Saúde, o termo é freqüentemente confundido com práticas de prevenção, educação e comunicação em saúde, não tendo sido de fato implementadas políticas e ações no cotidiano dos serviços, apesar dos avanços em termos de legislação e do respaldo obtido nas conferências de saúde.

Mas, para que a comunidade tenha atuação e participação ativa nas políticas de saúde, devem ser fornecidos meios para sua capacitação, como apoio de profissionais da saúde, da educação e de outros setores e de ONGs, a fim de que a população seja capaz de levantar discussões sobre os problemas que a afetam, fazendo conexão entre saúde e condições de vida e possibilitando "a construção de um novo saber, sem o qual o trabalho em saúde não alcança os objetivos desejados - as doenças continuam atingindo a população, fazendonos perguntar qual a eficácia e finalidade de nossa atuação como profissionais em defesa da saúde e da vida"5.

Há necessidade de incremento sociocultural da comunidade para que esta seja participativa e crítica, e exerça a cidadania, pois se constata reiterada e ostensiva inobservância dos direitos de cidadania contra a maioria da população, excluída dos bens e serviços desfrutados pelas elites. Segundo Bodstein6, "cidadania, democracia e direitos, mesmo quando confrontados com desigualdade, miséria e exclusão social, revelam o caminho possível da luta política e da busca de autonomia", num processo que "certamente implica o fortalecimento da sociedade civil e de suas formas plurais de organização". Essa mesma autora continua: "É através do exercício da cidadania que massas amorfas (ou a sociedade fragmentada) podem constituir novos vínculos e identidades sociais, redefinindo formas inovadoras de solidariedade".

Nos países em desenvolvimento, a cegueira é reconhecida como problema de saúde pública7 ${ }^{7}$. Cerca de $20 \%$ das crianças em idade escolar apresentam algum transtorno da visão, sendo comuns os distúrbios visuais nas crianças carentes ${ }^{8}$. As causas mais freqüentes de acuidade visual reduzida em escolares são os erros de refração, estrabismo e ambliopia ${ }^{9}$, sendo que pelo menos $60 \%$ das causas de cegueira e severo comprometimento visual infantil são preveníveis ou tratáveis ${ }^{10}$.

As deficiências visuais interferem no processo de aprendizagem e no desenvolvimento psicossocial da criança ${ }^{9}$, uma vez que, segundo Mansini em Lucas et al.11, "a visão é a grande promotora da integração das atividades motora, perceptiva e mental", havendo necessidade de detecção precoce das deficiências visuais, antes da idade de sete $\operatorname{anos}^{12}$, a fim de garantir integração das crianças na sociedade.

\section{OBJETIVOS}

Promover a capacitação de alunos de Medicina da disciplina de Saúde Coletiva da Universidade Federal do Amazonas (Ufam) e comunidade, preparando o aluno para o futuro exercício de sua profissão e contribuindo para a garantia do direito a cidades saudáveis através do exercício pleno da cidadania, baseado no desenvolvimento de uma cultura cidadã dentro do paradigma socioecológico.

Objetivou-se também identificar os problemas oftalmológicos prevalentes em escolares da comunidade e orientar o tratamento deles, tendo-se em vista a integração de conhecimentos pelos alunos, o estímulo à atividade preventista e a sensibilização da população para as questões referentes a sua saúde. 
De maneira mais específica, buscou-se o entendimento por parte dos alunos do papel da comunidade como sujeito e não como objeto das ações de saúde; estimular a prática de atividades preventistas pelos alunos; estimular atividades intersetoriais e estabelecimento de parcerias com vistas à Promoção da Saúde; contribuir para a democratização do conhecimento do processo saúde-doença e para o entendimento das relações políticas e sociais que envolvem a comunidade; e estimular a organização da comunidade para atuar na melhoria da qualidade de vida e saúde, com maior participação no controle desse processo.

\section{METODOLOGIA}

O estudo foi realizado numa comunidade na área urbana da periferia do município de Manaus, Amazonas, no bairro Colônia Antônio Aleixo, antigo leprosário, desativado em 1978, com uma população atual estimada de 23 mil habitantes. O bairro é dividido em seis áreas, sendo o trabalho desenvolvido numa delas, o conjunto Guilherme Alexandre e suas adjacências. O bairro é carente em prestação de serviços públicos, como bancos, correios e escolas com ensino fundamental. Com relação ao setor de saúde, o bairro é assistido por um hospital geral, uma maternidade e três centros de saúde, sendo um destes centros localizado no conjunto Guilherme Alexandre.

Os acadêmicos de Medicina envolvidos no estudo eram alunos do $5^{\mathrm{o}}$ ano deste curso cumprindo regularmente a disciplina obrigatória de Saúde Coletiva e corresponderam a 16 alunos, ou seja, $45 \%$ dos alunos matriculados nesta disciplina, divididos em dois grupos segundo a localidade de atuação e docentes. Os 16 alunos participantes foram, por sua vez, divididos em dois grupos de oito alunos, sendo que cada grupo se deslocava uma vez por semana para a comunidade sob a orientação do docente para realizar as atividades. Também uma vez por semana, alunos e professor se encontravam na Faculdade de Ciências da Saúde da Ufam para discutir temas pertinentes ao aprendizado da disciplina, ocasião em que todos comentavam o assunto e eram avaliados quanto ao nível de entendimento e conhecimentos adquiridos no decorrer do curso. No final deste, cada aluno devia apresentar um relatório das atividades na comunidade. No presente trabalho, são referidos os resultados das atividades de nove alunos.

As atividades foram desenvolvidas de setembro de 2002 a janeiro de 2003. Foram realizadas visitas domiciliares semanais, discutindo-se questões relevantes para a saúde dos moradores e problemas da localidade, que foram motivo de reuniões, promovendo-se a intersetorialidade com convidados cujo trabalho se relacionava aos problemas identificados. Além destas reuniões, também havia encontros semanais entre moradores da comunidade, voluntários e os integrantes do projeto, nos quais eram discutidos os rumos de abordagem deste trabalho, com sugestões oriundas da própria comunidade, e eram debatidos temas relacionados à saúde, qualidade de vida e educação em saúde.

A avaliação oftalmológica foi realizada em escolares de 4 a 14 anos e constou de duas partes. Numa primeira etapa, foi avaliada a acuidade visual por meio da tabela de Snellen a 5 metros do observador e avaliação de estrabismos por meio do teste de Hirschberg e do cover test; além disso, houve inspeção dos olhos e anexos, feita por acadêmicos de Medicina nas casas dos moradores da comunidade, observando-se sempre a correta realização dos testes com iluminação e ambiente adequados. Numa segunda etapa, os indivíduos com alterações eram encaminhados ao especialista para avaliação e tratamento.

Os critérios utilizados para encaminhamento ao oftalmologista foram baseados nos trabalhos de José \& Temporini ${ }^{13} \mathrm{e}$ Lopes et al.9: diminuição da acuidade visual (AV), tendo-se como limite AV £ 0,7, considerado conveniente em Oftalmologia Sanitária, ou diferença entre os dois olhos maior que duas linhas de optotipos; estrabismo; e alterações verificadas à inspeção como opacidade corneana e implantação incorreta dos cílios.

Durante a realização da avaliação oftalmológica, os familiares das crianças eram estimulados a interagir no exame e eram discutidos saúde ocular e o funcionamento do Sistema de Saúde no que tange ao aspecto oftalmológico.

\section{RESULTADOS}

Durante o projeto, foram visitados 112 domicílios, incluindo um total de 490 moradores, observando-se o predomínio de uma população de crianças e adultos jovens, com poucos idosos, sendo que 259 (52,8\%) indivíduos tinham menos de 20 anos. Verificou-se discreto predomínio de mulheres, que representaram 50,8\% da população total.

Com relação à situação de trabalho, das 207 pessoas que compuseram a população economicamente ativa, 49,3\% (102 indivíduos) se encontravam desempregados e 50,7\% (105 indivíduos) empregados, sendo a maioria na informalidade, com $72(68,6 \%)$ dos indivíduos sem carteira assinada e $33(31,4 \%)$ com carteira assinada.

A análise da renda mensal das famílias revelou que a grande maioria - 85 famílias $(75,9 \%)$ - recebia até 2 salários mínimos, sendo que $65,2 \%$ recebiam entre 1 e 2 salários, com apenas $24,1 \%$ recebendo mais de 2 salários mínimos. 
A análise da escolaridade da população em idade escolar revelou que 30 pessoas $(7,5 \%)$ não são alfabetizadas. Das alfabetizadas (371 pessoas), a maioria não chegou a completar o ensino fundamental, correspondendo a 272 indivíduos (67,8\%). Os indivíduos que chegaram a completar o ensino fundamental corresponderam a 15,2\% (61 pessoas) da população em idade escolar. Apenas 38 indivíduos (9,5\%) tinham o ensino médio completo.

Assim, a partir dos encontros entre os acadêmicos de Medicina e comunitários, pôde-se identificar os principais problemas que afetavam a comunidade:

- Desconhecimento das leis pelos moradores;

- Segurança pública inadequada às necessidades da comunidade;

- Serviço de saneamento básico precário;

- Desemprego e subemprego, que, levando ao ócio e à baixa renda, contribuíam para aumentar os problemas sociais da comunidade, como violência urbana, alcoolismo, criminalidade e abuso de drogas;

- Crianças em situação de risco social;

- Baixo grau de escolaridade dos moradores;

- Falta de serviços de assistência na comunidade, como bancos, correios, áreas de lazer, instituições de ensino e assistência à saúde, o que faz com que os moradores tenham de percorrer longas distâncias para ter acesso a tais serviços, por estradas de má qualidade e com transporte urbano de alto custo (ônibus).

Estes temas serviram de estímulo à realização de reuniões entre moradores, acadêmicos de Medicina e profissionais dos setores relacionados para discussão de problemas e soluções, promovendo, assim, a intersetorialidade. Os profissionais que participaram foram policiais civil e militar, auditora fiscal do trabalho, representantes do INSS, da Secretaria Municipal de Obras e Saneamento Básico, e do Departamento Municipal de Limpeza Pública. Estas reuniões geralmente eram realizadas na casa de moradores ou em outros locais na comunidade. Nestas, os elementos da comunidade eram estimulados e tinham a oportunidade de apresentar seus problemas e fazer questionamentos diretamente a profissionais que pudessem orientá-los em direção a uma solução. Muitas vezes, eram iniciadas medidas em busca de mudanças, com iniciativa e organização dos moradores. Nessas reuniões, a comunidade elaborou documentos com reivindicações a órgãos e autoridades políticas responsáveis. Foram também formados grupos de trabalho para a melhoria da segurança pública e a diminuição do desemprego.

Buscando-se estabelecer parcerias para o enfrentamento de problemas, durante este trabalho ocorreu a integração de atividades entre comunidade local, Departamento de Saúde Coletiva da Ufam, Instituto de Oftalmologia de Manaus, Funasa - que realiza cloração e fluoretação da água da localidade - e a ONG Morhan (Movimento de Reintegração do Hanseniano). Tentou-se uma parceria com o centro de saúde local a fim de possibilitar a inserção dos alunos no sistema de saúde municipal, mas não se conseguiu por falta de colaboração da direção desse centro.

Com relação à avaliação oftalmológica, 133 crianças de 4 a 14 anos de idade foram examinadas em suas próprias casas. Dessas, 37\% tinham entre 4 e 7 anos. Das 133 crianças, somente $10(7 \%)$ apresentaram algum tipo de alteração - diminuição da acuidade visual no teste de triagem na comunidade em todas elas - e foram, então, encaminhadas ao especialista. A avaliação especializada constatou que apenas uma das crianças encaminhadas precisava de tratamento, apresentando miopia, estando os 9 escolares restantes dentro dos limites da normalidade.

\section{DISCUSSÃO}

A integração entre universidade e comunidade, possibilitando ao aluno de Medicina ter como campo de atividades o próprio meio onde irá trabalhar, lidando com situações usuais da prática médica, aumenta o potencial de aprendizado dos acadêmicos, o que foi verificado nesta experiência, que se contrapõe ao modelo pedagógico vigente na Ufam, com ensino fragmentado e distante da realidade da comunidade. No decorrer do trabalho, os alunos se mostraram capazes de buscar soluções para as dificuldades encontradas na realização das atividades, construindo, assim, o conhecimento e agindo de maneira criativa diante de problemas comumente verificados nas comunidades. É necessário, porém, ressalvar o número de acadêmicos de Medicina envolvidos neste relato (nove alunos), de forma que a precisão das ilações aqui apresentadas poderia ser maior se mais alunos fossem incluídos neste trabalho.

O ensino de Medicina voltado de forma inovadora às necessidades sociais oriundas da comunidade permite que a escola médica se insira no SUS e forme profissionais e cidadãos com olhar crítico em relação à diferença entre saúde e serviços de saúde ${ }^{14}$, capazes de participar da construção de uma cidade saudável e da Promoção da Saúde.

Assim, o acadêmico de Medicina, inserido na realidade que o cerca, na comunidade na qual irá trabalhar, participa de um processo de capacitação mútua, na qual "o trabalho acadêmico fornece insumos que favorecem o crescimento da autonomia e iniciativa do movimento popular, ao mesmo tempo que a qualificação progressiva dos profissionais, cuja for- 
mação tem por tendência geral ignorar conhecimentos relevantes da questão saúde, ainda não incluídos nos currículos das escolas e universidades"

Sobre a interação entre acadêmico e comunidade, Valla ${ }^{5}$ diz que a mesma proporciona "a democratização do saber, chave para a construção de um novo saber, decodificado e reproduzido coletivamente e que pode se transformar em ações objetivas e reais voltadas para a modificação das condições de vida, com conhecimento e exercício pleno dos direitos e deveres dos cidadãos, neles incluídos os direitos à saúde". Esta observação de Valla é corroborada pelo que se pôde verificar neste trabalho, no qual a população desinformada encontrava meios para alcançar seus objetivos na medida em que tomava conhecimento das leis, relações sociais e serviços que a assistem, adequando a sua realidade conceitos e orientações em saúde e desenvolvendo a cidadania.

Desta forma, a comunidade foi fortalecida em suas lutas sociais e motivada a se organizar em busca de melhor qualidade de vida e exercício pleno da cidadania, sendo capaz de discutir os problemas que a afetam e fazendo conexão entre saúde e condições de vida. Ressalte-se, porém, a importância de trabalho contínuo com a comunidade, cujos resultados exigem paciência para que sejam atingidos.

Partindo-se da questão dos problemas de saúde da população, vê-se a necessidade de uma abordagem interdisciplinar e intersetorial como forma de superar ou melhorar as diversas situações que afetam a comunidade. O estabelecimento de parcerias é possível e necessário para o enfrentamento de problemas. A busca de melhor qualidade de vida como objetivo das ações de Promoção da Saúde passa por caminhos que incluem a integração de uma diversidade de profissionais e instituições atuando de forma conjunta e integrada ${ }^{4}$.

A informalidade, o subemprego, a baixa renda das famílias e o baixo grau de escolaridade privam as pessoas de direitos sociais conquistados. A baixa renda verificada está em acordo com o restante do Brasil, onde se observa que $65 \%$ das pessoas que trabalham recebem até um salário mínimo ${ }^{15}$, e é conseqüência da concentração de renda que ocorre no Amazonas quando se considera que o PIB per capita do estado era o sétimo maior do País (R\$7.169,00), segundo dados do IBGE em 2001. Os baixos salários são insuficientes para a manutenção das necessidades básicas da vida, sendo verificado em 2002 que a cesta básica mais barata encontrada em supermercados de Manaus levava mais da metade do salário mínimo de $\mathrm{R} \$ 200,00$, revelando a necessidade de um salário mínimo mais justo e adequado à realidade de uma família.

Com relação à rede de assistência à saúde na localidade, apesar de a comunidade ser assistida por um hospital geral, uma maternidade e três centros de saúde, os serviços de assistência à saúde têm organização e funcionalidade precárias, com baixa resolutividade e não atendem aos problemas prioritários da comunidade.

A respeito da abordagem oftalmológica realizada neste trabalho, observa-se que, apesar dos diversos problemas de saúde apresentados pelas populações carentes, ela possibilitou um caminho para os acadêmicos agirem de modo preventista e não curativo. Mas, contrastando com o que seria esperado de uma população carente, a prevalência de problemas oftalmológicos nos escolares da comunidade se mostrou inferior ao observado na literatura $8,9,16$.

A abordagem oftalmológica realizada na comunidade atuou também como uma forma de aumentar a auto-estima dos indivíduos, estimulando sua participação no controle do processo de melhoria de sua qualidade de vida e saúde. População e acadêmicos de Medicina foram, assim, apresentados à realidade do atendimento oftalmológico na localidade, gerando senso crítico e possibilitando também aos alunos a integração e associação dos conhecimentos, fragmentados em diferentes disciplinas no curso médico, uma vez que a promoção e prevenção da saúde ocular não é uma prática freqüente de profissionais de saúde no atendimento à população pediátrica, conforme observado por Sperandio ${ }^{12}$ ao estudar uma equipe de enfermeiros e pediatras vinculados aos serviços de saúde pública.

\section{CONSIDERAÇÕES FINAIS}

$\mathrm{O}$ aluno de Medicina, quando inserido na realidade que o cerca, tem maior potencial para ampliar, integrar e fazer intercâmbio de conhecimentos para o futuro exercício de sua profissão, capacitando a comunidade e tornando-se um profissional mais ativo na sociedade na luta por uma localidade saudável/cidade saudável.

Verificou-se também que as atividades realizadas com a comunidade e as discussões levantadas, resultado da interação acadêmico/comunidade, permitiram fortalecer e organizar a comunidade em suas lutas por melhor qualidade de vida e de saúde, propiciando uma participando mais ativa no controle desse processo.

Quanto à avaliação oftalmológica, observou-se que os problemas oftalmológicos em escolares no Conjunto Guilherme Alexandre são pouco prevalentes, contrariando os ensinamentos intramuros. Identificou-se também que a abordagem oftalmológica realizada tem um importante papel de sensibilização da comunidade para os problemas de saúde que a afetam.

Assim, sugere-se que os projetos pedagógicos das escolas médicas permitam a inserção precoce do aluno de Medicina 
na realidade que o cerca, adequando a escola médica ao Sistema de Saúde.

Ressalta-se também que a atuação da escola médica na comunidade deve se dar de maneira contínua e permanente, não apenas uma intervenção em curto período de tempo, a fim de que se possa observar os resultados das mudanças almejadas, que se dão em longo prazo.

Embora a prevalência de problemas oftalmológicos na população estudada tenha sido baixa, são importantes a manutenção e a implementação de medidas de promoção da saúde ocular na população infantil, como a Campanha Olho no Olho, realizada pelo Ministério da Educação e Conselho Brasileiro de Oftalmologia.

\section{REFERÊNCIAS}

1. Mendes EV. A evolução histórica da prática médica: suas implicações no ensino, na pesquisa e na tecnologia médicas. Belo Horizonte (MG): PUC-MG/FINEP; 1984.

2. Brasil. Ministério da Saúde. Promoção da Saúde: Carta de Ottawa, Declaração de Adelaide, Sundsvall e Santa Fé de Bogotá. Brasília (DF): Ministério da Saúde; 1996.

3. Mello DA, Rouquayrol MZ, Araújo C et al. Promoção à saúde e educação: diagnóstico de saneamento através da pesquisa participante articulada à educação popular (Distrito São João dos Queiróz, Quixadá, Ceará, Brasil). Cad Saúde Pública. 1998; 14 (3).

4. Mello DA. Reflexões sobre promoção à saúde no contexto do Brasil. Cad Saúde Pública. 2000; 16(4).

5. Valla VV, Carvalho M. Participação popular e os serviços de saúde. O controle social como exercício da cidadania. Rio de Janeiro: Fundação Oswaldo Cruz, Escola Nacional de Saúde Pública; 1993.

6. Bodstein RCA. Cidadania e modernidade: emergência da questão social na agenda pública. Cad Saúde Pública. 1997; $1.13(2)$.

7. Monteiro ECL, Pereira TCS, Schellini AS, Silva MRBM, Padovani CR. Alterações oculares em pré-escolares em Botucatu, SP. Pediatria Atual. 1999; 12 (3).

8. Albuquerque RC, Alves JGB. Afecções oculares prevalentes em crianças de baixa renda atendidas em um serviço oftalmológico na cidade do Recife - PE, Brasil. Arq Bras Oftalmol. 2003; 66(6).
9. Lopes GJA, Casella AMB, Chuí CA. Prevalência de acuidade visual reduzida nos alunos da primeira série do ensino fundamental das redes pública estadual e privada de Londrina-PR, no ano de 2000. Arq Bras Oftalmol. 2002; 65(6).

10. Brito PR, Veitzman S. Causas de cegueira e baixa visão em crianças. Arq Bras Oftalmol. 2000; 63(1).

11. Lucas MB, Leal DB, Tavares SS, Barros EA, Aranha ST. Condutas reabilitacionais em pacientes com baixa visão. Arq Bras Oftalmol. 2003; 66(1).

12. Sperandio AMG. Promoção da saúde ocular e prevenção precoce de problemas visuais nos serviços de saúde pública. Rev. Saúde Públ. 1999. out. vol. 33 n. 5.

13. José NK, Temporini ER. Avaliação dos critérios de triagem visual de escolares de primeira série do primeiro grau. Rev Saúde Publica. 1980; 14: 205-14.

14. Vieira JE. Definição de necessidades sociais para o ensino médico. Rev Bras Educ Med. 2003; 27(2):153-157.

15. Dias EC. Aspectos atuais da saúde do trabalhador. In: Buschnelle J, Rocha L, Rigotto R. Isto é trabalho de gente? Vida, doença e trabalho no Brasil. Petrópolis (RJ): Vozes; 1994. p.138-56.

16. Schimiti RB, Costa VP, Gregui MJF, Kara-José N, Temporini ER. Prevalence of refractive errors and ocular disorders in preschool and school children of Ibiporã - PR, Brazil (1989 to 1996). Arq Bras Oftalmol. 2001; 64(5).

\section{Conflito de Interesses}

Os autores do artigo "Promoção da Saúde e Cultura Cidadã Envolvendo uma Abordagem Oftalmológica em Escolares na Colônia Antônio Aleixo (CAA), Manaus - AM: Uma Experiência no Ensino Médico" declaram a inexistência de algum tipo de Conflito de Interesse durante a elaboração deste trabalho.

\section{Endereço para correspondência}

Jefferson Augusto Santana Ribeiro

Conjunto Adrianópolis - quadra N - casa 01

Bairro Adrianópolis

69060-490 - Manaus - Amazonas

E-mail: jef_ribeiro@yahoo.com.br 